\title{
Identidad vocacional, claridad del autoconcepto y autoestima en adolescentes peruanos
}

\author{
Joaquín Castro Valdez* \\ Proyecto Suma: Asistencia y Rehabilitación en Salud Mental, Argentina
}

(Recibido 27 Septiembre, 2014; Aceptado 8 Febrero, 2015)

RESUMEN: La decisión vocacional representa una de las tareas fundamentales del final de la adolescencia, y como tal, está involucrada directamente con los distintos aspectos del sí mismo. El objetivo de este estudio ha sido describir la relación entre las variables de autoestima y claridad del autoconcepto, como aspectos del autoconcepto, y la exploración y el compromiso como componentes de la identidad vocacional, en 182 estudiantes adolescentes de cuarto y quinto de secundaria. Con dicho fin, se aplicó el Cuestionario de Desarrollo de Carrera, la Escala de Identidad Vocacional, la Escala de Autoestima de Rosenberg, y la Escala de Claridad del Autoconcepto. Se hallaron correlaciones significativas medianas y grandes entre las cuatro variables, y se observaron diferencias de acuerdo al sexo y el grado de estudios. Usando correlaciones parciales para controlar el efecto de las variables del autoconcepto, se encontró que solamente la Claridad del Autoconcepto correlaciona significativamente con Compromiso, y solamente Autoestima correlaciona significativamente con Exploración. Las correlaciones parciales se mantuvieron similares en la muestra dividida por sexo, pero se observaron diferencias en la muestra dividida por grado de estudios.

Palabras Clave: identidad vocacional, exploración, compromiso, claridad del autoconcepto, autoestima.

\section{Vocational identity, self-concept clarity and self-esteem in Peruvian ado- lescents}

ABSTRACT: Vocational choice represents a fundamental task in late adolescence and, as such, it is directly involved with the multiple aspects of the self. The aim of this study was to describe the relationship between the variables of Self-Esteem and Self-Concept Clarity, as aspects of Self-Concept, and Exploration and Commitment as components of Vocational Identity, in 182 adolescent students in the last two years of high school education. Those variables were assessed through the Career Development Inventory, the Vocational Identity Scale, the Rosenberg Self-esteem Scale, and the Self-concept Clarity Scale. Significant correlations were found between the four variables, and differences were observed according to gender and level of education. Using partial correlations to control the effect of the variables of the self-concept, it was found that only Self-Concept Clarity had a significant correlation with Commitment, and only Self-Esteem had a significant correlation with Exploration. Partial correlations remained similar in the sample in terms of gender, but differences were observed 
in terms of level of education.

Keywords: vocational identity, exploration, commitment, self-concept clarity, self-esteem.

\section{INTRODUCCIÓN}

La consolidación de la vocación y de la carrera son piezas fundamentales en el proceso de formación de la identidad adulta, que constituye el final de la etapa adolescente (Erikson 1974, 2000). La decisión vocacional, si bien es un proceso que se desarrolla a través de toda la vida, es considerada como una de las principales tareas del desarrollo del adolescente (Baumeister y Twenge, 2003; Erikson, 1974; López, 2003; Macovei, 2009; Skorikov, 2006).

Siendo un proceso crucial en la formación de la identidad, y enmarcado al mismo tiempo en la transición del colegio a la educación superior, la elección de una carrera puede además estar marcada por una gran carga de estrés, aumentada a su vez por una situación de conflicto entre las preferencias y las posibilidades, entre exigencias externas y aspiraciones propias, el miedo al fracaso y la voluntad de logro, entre otras, por lo que el período de decisión puede resultar ser particularmente difícil (Collins y Laursen, 2004; Florenzano, 1993; Hirschi, 2012; Savickas, 1990). La capacidad de un adolescente para optar por una carrera en un momento determinado dependerá de una serie de características de sí mismo (Hisrchi, 2010, 2008; Holland, 1959; Stringer y Kelperman, 2010). Resulta, por ende, indispensable el conocimiento adecuado sobre este proceso, que resulte en la posibilidad de brindar acompañamiento y orientación acorde a las particularidades de cada persona.

\section{Identidad vocacional}

El constructo de identidad vocacional ha sido estudiado en gran medida desde la conceptualización de Holland (1996), que lo propone como una noción clara de las metas, intereses, habilidades y posibilidades ocupacionales de uno mismo. Esta noción es el fruto de un proceso de creciente diferenciación durante la niñez y la adolescencia respecto a las otras personas en cuanto a intereses, competencias y valores (Holland, 1985 citado por Vondracek, 1992). Holland señala como fundamental estudiar la medida en que una persona se distingue de las demás (diferenciación), cuán relacionados entre sí están sus intereses vocacionales (consistencia de intereses), y la cercanía de sus intereses con su trabajo real (congruencia), postulando que debería hallarse una relación positiva entre estas medidas y una identidad vocacional más desarrollada (Hirschi, 2011a; Holland, 1996).

No obstante, diversos estudios empíricos han mostrado resultados inconsistentes en relación a esa hipótesis (Hirschi y Läge, 2007; Vondracek y Skorikov, 1997; véase también Hirschi, 2011a). Distintos investigadores sostienen que se debe a que es una conceptualización relativamente simplista, que sólo considera el compromiso con las decisiones y las nociones claras sobre uno mismo, dejan- 
do de lado la importancia de la exploración de distintas posibilidades (Vondracek, 1992; Hirschi, 2011c).

Por otro lado, tenemos el modelo teórico de la identidad propuesto por Marcia $(1966,1980)$, quien operacionaliza el constructo ampliamente trabajado por Erikson, planteando la noción de identidad como una organización interna, autoconstruida y dinámica de impulsos, habilidades, creencias e historia individual, que varía gradualmente de acuerdo a la edad y a las experiencias del individuo, y que se encuentra en constante desarrollo. Para este autor, la capacidad para realizar de forma satisfactoria una elección importante se encuentra relacionada con un período de crisis en que se da la exploración de las alternativas existentes durante una etapa de toma de decisiones, y el compromiso con la opción u opciones escogidas, entendida como el hecho de optar por una alternativa y adherirse a esa elección (Marcia, 1967; Vondracek, Schulenberg, Skorikov, Gillespie, y Wahlheim, 1995).

Este modelo introduce una tipificación de estatus de identidad, proponiendo cuatro estatus independientes característicos de la adolescencia: moratoria, si se demora la elección pero existe exploración (exploración alta, compromiso bajo); cerrada, cuando el individuo ha realizado su elección sin explorar otras opciones (exploración baja, compromiso alto); lograda, si es capaz de realizar una elección en función a la información que ha explorado (exploración y compromiso altos); y difusa, cuando la persona no tiene una dirección clara, pero tampoco se involucra en tareas de exploración (exploración y compromiso bajos) (Jara, 2010; Marcia, 1980).

La propuesta teórica de Marcia sigue siendo hasta el día de hoy uno de los modelos más importantes y más utilizados para la identificación y medición del constructo de identidad, y particularmente de identidad vocacional, principalmente en la adolescencia tardía (Hirschi, 2011a, 2011b, 2011c; Luyckx, Goossens, Soenens, Beyers, y Vansteenkiste, 2005; Schwartz, 2001; Vondracek, 1992; Vondracek y Skorikov, 1997; Waterman, 1988).

\section{Autoconcepto}

Dentro de los múltiples factores implicados en la elección de una carrera, y por ende en la conformación de la identidad vocacional, los factores internos del individuo son los que ocupan un mayor interés para la investigación académica en Psicología, y así también en el presente estudio. En este grupo se halla el autoconcepto, como un factor propio del sí-mismo.

El autoconcepto es definido actualmente como un esquema cognitivo, organizado y estructurado, que contiene la totalidad de las creencias de un individuo sobre sus propias características, así como también roles, valores y metas personales (Campbell, 1990; Campbell et al., 1996; Steffgen, Da Silva, Recchia, 2007; Stinson, Wood, Doxey, 2008). Uno de los constructos más investigados dentro de este esquema es la autoestima, que supone una parte de la dimensión afectivoevaluativa del autoconcepto (Campbell, 1990; Fleming y Courtney, 1984). 
A través de los años, diversas investigaciones han hecho esfuerzos por estudiar características evaluativas del autoconcepto de los individuos como un factor influyente en relación a su identidad vocacional. Si bien una buena cantidad de estudios realizados en adolescentes, tanto en etapa escolar como en los primeros años de estudios superiores, han encontrado que una autoestima más positiva se encuentra relacionada con una identidad vocacional más desarrollada y estable (destacamos a Barret y Tinsley, 1977; Malone, 1989; Munson, 1992; Patton, Bartrum, y Creed, 2004), otras investigaciones no han podido encontrar evidencias significativas que sustenten dicha relación (Bedeian, 1977; Lawrence y Brown, 1976; Rehberg y Hotchkiss, 1979), lo que hace que los hallazgos de las investigaciones resulten ciertamente inconsistentes.

En ese sentido, la falta de investigación en relación al rol que la dimensión estructural del autoconcepto representa con respecto al proceso de elección de una carrera, constituye un gran vacío en la investigación sobre identidad vocacional, si bien diversos autores han sugerido la importancia que tendrían los componentes estructurales del autoconcepto como predictores en la elección de la carrera. En esta línea, encontramos investigaciones que han asociado aspectos de la identidad vocacional con constructos relacionados a la estabilidad y a componentes estructurales del funcionamiento psicológico, como estabilidad de metas (Robbins y Patton, 1985; Santos, 2003; Strauser, Lustig, y Çiftçi, 2008), estabilidad de intereses (Tracey y Robbins, 2005; Tracey, Robbins, y Hofsess, 2005; Mullis, Mullis, y Gerwel, 1998; Rottinghaus, 2007; Swanson, 1999; Lubinski, Benbow, y Ryan, 1995; King, 1958), y el logro de la identidad del yo (Savickas, 1985) obteniendo resultados favorables.

De este modo, el presente estudio ha tenido como objetivo la investigación de la relación que tiene el aspecto estructural del autoconcepto con la identidad vocacional, en comparación con el aspecto afectivo-evaluativo.

Este aspecto estructural del autoconcepto se estudió a partir del constructo de claridad del autoconcepto, el cual es definido como "la medida en que los contenidos del autoconcepto de un individuo (por ejemplo, los atributos personales percibidos) son definidos con claridad y confianza, poseen coherencia interna, y son temporalmente estables" (Campbell et al., 1996, p. 141). La claridad del autoconcepto representa un indicador de ajuste, en tanto previene problemas sociales, emocionales y motivacionales derivados de la incertidumbre acerca de uno mismo (Baumeister, 1986; Brown y Smart, 1989, ambos en Campbell, 1990; Campbell, 2003), y se relaciona con estrategias de afrontamiento activas (Smith y Dust, 2006; Smith, Wethington, y Zhan, 1996, en Demirutku, 2007).

Considerando lo expuesto, el estudio se enfocó en la relación entre el aspecto estructural y el afectivo del autoconcepto, a través de los constructos de autoestima y claridad del autoconcepto respectivamente, y los dos componentes de la identidad vocacional, la exploración y el compromiso. Se otorgó especial interés a la manera en que se asocian las dos primeras variables con cada uno de los componentes de la identidad vocacional. La visión de esta investigación fue aportar una mirada distinta al estudio de la identidad vocacional, debido a que 
no se han encontrado otros estudios que la relacionen con la claridad del autoconcepto, que podría ser un constructo muy valioso para el entendimiento de la orientación hacia la elección vocacional.

Teniendo en cuenta que hay buena cantidad de investigaciones que sugieren que el sexo del individuo implica diferencias para la forma en que se presentan los componentes del autoconcepto (Castañeda, 2013; Gurung, R. A., Sarason, B. R., y Sarason, I. G., 2001; Josephs y Markus.,1992; Reina, Oliva, y Parra, 2010; Suh, 2002) y de la identidad vocacional (Gushue, Clarke, Pantzer, y Scanlan, 2006; Hirschi, 2011a, 2011c; Munson, 1992; Patton et al., 2004), se exploró cómo se hallan dichas variables en esta muestra en función al sexo, como objetivo adicional. Asimismo, existen estudios (Holland, Daiger et al., 1980; Kalakoski y Nurmi, 1998; Niece y Bradley, 1979; Santos, 2003) que apuntan a que tanto la edad como el grado de estudios y la etapa particular en la que se encuentran los individuos, influyen en los componentes de la identidad vocacional. Por otro lado, en el contexto particular en que se lleva a cabo la presente investigación, típicamente durante el último año de secundaria se dan las postulaciones a los estudios superiores (universidades e institutos), lo que supone una diferencia importante entre los dos últimos años de estudios escolares y que debería ser abordada. Teniendo lo anterior en consideración, se explorará también como un objetivo adicional las variables en función de la edad y grado de estudios.

\section{MÉTODO}

\section{Participantes}

Participaron 182 estudiantes de un colegio particular de la ciudad de Lima, entre los 14 y 18 años de edad ( $M=15.9, S D=0.794,44 \%$ mujeres), de cuarto grado ( $N=91$, edades de 14 a 18 años, $M=15.49, S D=0.766 ; 42 \%$ mujeres) y quinto grado $(N=91$, edades de 15 a 18 años, $M=16.31, S D=0.591 ; 46 \%$ mujeres) de secundaria. Dicha institución es ampliamente reconocida por el énfasis que otorga a la importancia de continuar los estudios académicos en instituciones de formación superior.

El acceso a la muestra se obtuvo a través del Departamento Psicopedagógico de dicha institución, y la aplicación del estudio se realizó de forma grupal y voluntaria en cuatro días distintos.

\section{Instrumentos}

\section{Exploración vocacional}

Se utilizó la versión en castellano de las sub-escalas "planificación" y “exploración", que constituyen la dimensión "actitudinal" del Cuestionario de desarrollo de carrera - CDC (Super et al., 1981), adaptado y validado por Álvarez González (1989; Álvarez González, Bisquerra, Espín, y Rodríguez Espinar, 2007). La sub-escala de exploración tiene 16 ítems, en los que se pregunta al evaluado si 
es que consultaría a distintas fuentes para obtener información sobre una carrera, y cuánta información útil ya ha obtenido de cada fuente (como padres, maestros, orientadores). La sub-escala de planificación tiene 17 ítems, y en ella se pide indicar la cantidad de tiempo utilizado y actividades realizadas para conocer más sobre alguna carrera. En ambas sub-escalas existen cinco opciones para cada enunciado (por ejemplo, desde "mis planes no son nada claros ni seguros, sino más bien confusos", hasta "están muy claros y decididos"), y en la calificación se le asigna de 0.25 a 1 punto a cada opción. Las sub-escalas se combinaron sumando el puntaje de los 33 ítems del instrumento. En el presente estudio obtuvo un alfa de Cronbach de 0.907.

\section{Compromiso vocacional}

Se utilizó la Escala de Identidad Vocacional, una sub-escala de la prueba Mi Situación Vocacional (Holland, Daiger, y Power, 1980), en su versión adaptada al castellano y validada por Tosado (2012). La escala es autoaplicada y consiste de 18 ítems dicotómicos (verdadero y falso), todos inversos. Mide la ausencia de dificultades en la toma de decisiones y la confianza en la propia habilidad para tomar decisiones en situaciones ambiguas (Munson, 1992); un puntaje alto indicará confianza en la capacidad de uno mismo para tomar decisiones, y un compromiso alto con las alternativas por las que se opte. Ha sido utilizada para evaluar el constructo de compromiso en diversas investigaciones (Hirschi, 2009, 2010, 2011a, 2011d; Hirschi y Läge, 2008), dado que mide el compromiso en la toma de decisiones tal como lo propone la tipificación de Marcia (1980). En el presente estudio obtuvo un alfa de Cronbach de 0.814 .

\section{Autoestima}

Se usó la versión en castellano de la Escala de Autoestima de Rosenberg (1965), traducida para la edición en castellano de Rosenberg (1973) y validada por Atienza, Moreno y Balaguer (2000). La escala es autoaplicada y consiste de 5 ítems directos $(1,2,4,6,7)$ y 5 inversos $(2,5,8,9,10)$, en formato tipo Lickert que va de 1 (muy en desacuerdo) a 4 puntos (muy de acuerdo). Se califica asignando el puntaje inverso a las afirmaciones direccionadas negativamente, y los valores teóricos fluctúan entre 10 (baja autoestima) y 40 (alta autoestima). Fue diseñada para evaluar la autoestima global de la persona a través de los sentimientos generales acerca de sí misma (Rosenberg, 1973). En el presente estudio obtuvo un alfa de Cronbach de 0.795 .

\section{Claridad del Autoconcepto}

Se utilizó la Escala de Claridad del Autoconcepto-SCC (Campbell et al., 1996), en su versión peruana por Argumedo y Romero (2010). La escala es autoaplicada y consta de 12 ítems en formato de tipo Lickert, que va desde 1 (fuertemente en desacuerdo) a 5 puntos (fuertemente de acuerdo). Evalúa la medida en que los contenidos del autoconcepto de una persona están claramente definidos, son internamente consistentes, y temporalmente estables. En el presente 
estudio obtuvo un alfa de Cronbach de 0.770 .

\section{Análisis estadístico}

Se calcularon los estadísticos descriptivos para la muestra completa y de acuerdo a las variables demográficas. La normalidad fue evaluada a través de la prueba de Kolmogorov-Smirnov con corrección de la significación de Lilliefors; dado que no se encontró una distribución normal en la mayoría de los instrumentos, se utilizó la prueba U de Mann-Whitney como estadístico para la comparación de medianas. Las correlaciones bivariadas se realizaron con la prueba rho de Spearman, debido a la naturaleza no paramétrica de los instrumentos descrita previamente, así como por el carácter ordinal de los puntajes (Martínez, Tuya, Martínez, Pérez, y Cánovas, 2009). Para calcular la proporción de las varianzas de Exploración y Compromiso explicada por la presencia de Claridad del Autoconcepto y Autoestima, se realizaron correlaciones parciales a través de la prueba $r$ de Pearson.

\section{RESULTADOS}

En la muestra completa, no se descartó la distribución normal para los puntajes de las medidas Claridad del Autoconcepto y Exploración, $D=.048, p=.200$, y $D=.058, p=.200$, respectivamente. No obstante, no se asumió la distribución normal para los puntajes de Autoestima y Compromiso, $D=0.74, p=.017$, y $D$ $=.101, p=.200$, respectivamente.

\section{Comparaciones a nivel descriptivo}

La Tabla 1 muestra que las poblaciones masculina y femenina mostraron diferencias significativas en la medida de Exploración; se observa un puntaje más elevado y significativamente mayor en el grupo femenino. Asimismo se observan diferencias significativas en el puntaje de Exploración, en que quinto de secundaria obtuvo un promedio más elevado comparado a cuarto de secundaria. Igualmente, el promedio obtenido por los participantes de quinto de secundaria fue significativamente mayor en la medida de Compromiso. Se exploraron otros grupos de la muestra sin encontrar diferencias significativas.

Tabla 1. Puntajes Promedio según Sexo y Grado de Estudios, y Coeficientes U de Mann-Whitney

\begin{tabular}{|c|c|c|c|c|c|c|c|c|c|c|c|c|c|c|}
\hline & \multicolumn{3}{|c|}{ Hombres } & \multicolumn{3}{|c|}{ Mujeres } & \multirow[b]{2}{*}{ U } & \multicolumn{3}{|c|}{ Cuarto } & \multicolumn{3}{|c|}{ Quinto } & \multirow[b]{2}{*}{$\mathrm{U}$} \\
\hline & $\mathrm{N}$ & $\mathrm{M}$ & $\mathrm{DE}$ & $\mathrm{N}$ & $\mathrm{M}$ & $\mathrm{DE}$ & & $\mathrm{N}$ & M & $\mathrm{DE}$ & $\mathrm{N}$ & M & $\mathrm{DE}$ & \\
\hline Claridad & 102 & 38 & 7 & 80 & 39 & 8 & $4.555,500$ & 91 & 38 & 8 & 91 & 39 & 7 & $4.159,000$ \\
\hline Autoestima & 102 & 31 & 5 & 80 & 31 & 4 & $4.159,500$ & 91 & 31 & 4 & 91 & 32 & 4 & $4.675,500$ \\
\hline Exploración & 102 & 111 & 22 & 80 & 117 & 17 & $4.812,000^{*}$ & 91 & 110 & 20 & 91 & 118 & 19 & $5.025,500^{*}$ \\
\hline Compromiso & 102 & 9 & 4 & 80 & 9 & 4 & $3.906,000$ & 91 & 8 & 4 & 91 & 10 & 4 & $4.924,500^{*}$ \\
\hline
\end{tabular}

$* \mathrm{p}<0,05$

$n=182$ 


\section{Correlaciones}

Como se presenta en la Tabla 2, el coeficiente más alto fue el de Claridad del Autoconcepto y Autoestima, una correlación grande de acuerdo al criterio de Cohen (1988), y similar en todos los grupos de la muestra. En la muestra completa, las cuatro variables obtuvieron correlaciones altamente significativas entre sí. Se observa una correlación mediana entre Compromiso y Exploración, Claridad del Autoconcepto y Compromiso, Autoestima y Compromiso, y Autoestima y Exploración. Entre las medidas de Claridad del Autoconcepto y de Exploración se observa una correlación pequeña, aunque igualmente significativa.

En la muestra diferenciada por sexo, la correlación entre Exploración y Compromiso fue mediana en hombres y grande en mujeres. Claridad del Autoconcepto mostró una correlación pequeña con Exploración en hombres, y ninguna en mujeres. En ambos grupos se registró una correlación mediana entre Claridad del Autoconcepto y Compromiso, mayor en hombres que en mujeres. La correlación entre Autoestima y Exploración fue pequeña en hombres y mediana en mujeres, y entre Autoestima y Compromiso fue mediana tanto en hombres como en mujeres, aunque mayor en mujeres.

En la muestra diferenciada por grado de estudios, se registró una correlación grande y altamente significativa entre Claridad del Autoconcepto y de Autoestima en ambos grados. Claridad del Autoconcepto mostró una correlación pequeña con Exploración en cuarto, y ninguna en quinto. En ambos grupos se observa una correlación mediana entre Claridad del Autoconcepto y Compromiso, siendo mayor en cuarto. La correlación entre Autoestima y Exploración fue pequeña en cuarto y mediana en quinto, y entre Autoestima y Compromiso fue mediana en cuarto y pequeña en quinto. La correlación entre Exploración y Compromiso fue mediana en ambos grados.

Tabla 2. Coeficientes de Correlación de Spearman entre Escalas, en la Muestra Completa y en Función a Sexo y Grado de Estudios

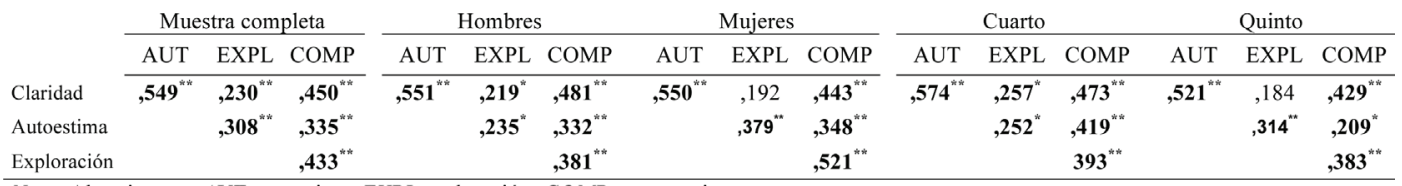

Nota: Abreviaturas: $A U T$ autoestima, EXPL exploración, COMP compromiso

${ }^{*} \mathrm{p}<0,05 ; * * \mathrm{p}<0,01$

$n=182$

\section{Correlaciones parciales}

En la muestra completa, controlando la variable Autoestima, Claridad del Autoconcepto obtuvo una correlación significativa y mediana con Compromiso, y controlando la variable Claridad del Autoconcepto, Autoestima obtuvo una correlación significativa y pequeña con Exploración. 
Tabla 3. Correlaciones Parciales en la Muestra Completa y en Función a Sexo y Grado de Estudios, Controlando Autoestima y Claridad del Autoconcepto

\begin{tabular}{|c|c|c|c|}
\hline & & $\begin{array}{l}\text { Controlada } \\
\text { Autoestima }\end{array}$ & $\begin{array}{c}\text { Controlada } \\
\text { Claridad }\end{array}$ \\
\hline Muestra completa & $\begin{array}{r}\text { EXPL } \\
\text { COMP }\end{array}$ & $\begin{array}{c}\text { CLA } \\
, 051 \\
, \mathbf{3 0 0 * *}\end{array}$ & 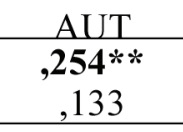 \\
\hline Hombres & $\begin{array}{r}\text { EXPL } \\
\text { COMP }\end{array}$ & $\begin{array}{c}, 047 \\
, \mathbf{3 0 4} * *\end{array}$ & $\begin{array}{l}, 245 * \\
, 165\end{array}$ \\
\hline Mujeres & $\begin{array}{r}\text { EXPL } \\
\text { COMP }\end{array}$ & $\begin{array}{c}, 034 \\
, 306 * *\end{array}$ & $\begin{array}{c}, 285 * \\
, 101 \\
\end{array}$ \\
\hline Cuarto & $\begin{array}{r}\text { EXPL } \\
\text { COMP }\end{array}$ & $\begin{array}{c}, 086 \\
, 272 * *\end{array}$ & $\begin{array}{l}, 234 * \\
, 245 *\end{array}$ \\
\hline Quinto & $\begin{array}{r}\text { EXPL } \\
\text { COMP }\end{array}$ & $\begin{array}{c}, 035 \\
, \mathbf{3 4 3} * *\end{array}$ & $\begin{array}{l}233 * \\
-, 011 \\
\end{array}$ \\
\hline
\end{tabular}

Nota: Abreviaturas: CLA claridad del autoconcepto, $A U T$ autoestima, EXPL exploración, COMP compromiso $* \mathrm{p}<0,05 ; * * \mathrm{p}<0,01$ $n=182$

Calculando el coeficiente de determinación $R^{2}$ es posible afirmar que el $9 \%$ de la varianza de Compromiso es únicamente explicada por la Claridad del Autoconcepto, y Autoestima no alcanza a explicar de forma significativa la varianza de Compromiso. Del mismo modo, el 6,45\% de la varianza de Exploración es únicamente explicada por la Autoestima, y Claridad del Autoconcepto no alcanza a explicar de forma significativa la varianza de Exploración.

En la muestra diferenciada por sexo los coeficientes fueron muy similares a los de la muestra completa. Al calcular $R^{2}$ en hombres, el 9,24\% de la varianza de Compromiso es únicamente explicada por la Claridad del Autoconcepto, y el 9,36\% en mujeres; en ambos grupos, Autoestima no alcanza a explicar de forma significativa la varianza de Compromiso. Del mismo modo, en hombres el 6\% de la varianza de Exploración es únicamente explicada por la Autoestima, y el $8,12 \%$ en mujeres; nuevamente, en ambos grupos Claridad del Autoconcepto no alcanza a explicar de forma significativa la varianza de Exploración.

En la muestra diferenciada por grado de estudios se hallaron diferencias importantes con respecto a la muestra completa. Controlando Autoestima, la correlación entre Claridad del Autoconcepto y Compromiso fue pequeña en cuarto grado y mediana en quinto. Del mismo modo, controlando la variable Claridad del Autoconcepto, Autoestima mostró correlaciones significativas y pequeñas con Exploración, y en cuarto mostró un coeficiente de correlación pequeño con Compromiso, notablemente diferente al observado en quinto. 
Calculando $R^{2}$ se observa que en cuarto el 7,40\% de la varianza de Compromiso es únicamente explicada por la Claridad del Autoconcepto, y en quinto el $11,76 \%$. En cuarto de secundaria el 6\% de la varianza de Compromiso es explicada únicamente por Autoestima, a diferencia de quinto, donde Autoestima no alcanzó a explicar de forma significativa la varianza de Compromiso. Asimismo, en cuarto el 5,48\% de la varianza de Exploración es únicamente explicada por la Autoestima, y en quinto es el 5,43 \%. En ambos grupos Claridad del Autoconcepto no llega a explicar de forma significativa la variabilidad de Exploración.

\section{DISCUSIÓN}

El hallazgo central para la presente investigación fue que, tanto en las correlaciones simples como en las parciales, la Claridad del Autoconcepto mostró una relación importante con el Compromiso, y la Autoestima con la Exploración. Si bien los análisis estadísticos utilizados no permiten hablar de relaciones causales, sobre la primera relación es posible señalar que una idea más clara, coherente y temporalmente estable de uno mismo permitirá también mostrar una noción estable, coherente y diferenciada de los propios intereses vocacionales, de lo que un individuo quiere ser y hacer.

Con respecto a la segunda relación, cabe sugerir que una Autoestima elevada puede significar mayor confianza en sí mismo para explorar diversas alternativas y buscar información de diversas fuentes, considerando los aportes externos sin temor a que confronten a las propias creencias y que se pueda encontrar información desfavorable. Del mismo modo una valoración negativa con respecto a sí mismo, llevaría al adolescente a no tener una base segura que le permita indagar en las alternativas de la realidad en base a sus propias capacidades. Por su parte, involucrarse en tareas de exploración tendría un efecto positivo en la autoestima del individuo, en tanto el hecho de estar realizando esfuerzos dirigidos a un mejor conocimiento sobre las propias capacidades y sobre su futuro, supone un afrontamiento activo del proceso de crisis propio de esta etapa del desarrollo, a través del esfuerzo de uno mismo por acercarse a lo que se quiere llegar a ser.

Respecto a las diferencias observadas en función del grado de estudios, es posible suponer que a medida que se avanza en edad y en grado de estudios, la relación del aspecto estructural del autoconcepto y la decisión sobre la carrera se vuelve más clara, y menos influida por la dimensión afectivo-evaluativa del autoconcepto. En esta línea, en tercero de secundaria y grados anteriores se esperaría encontrar una relación importante entre el componente afectivo-evaluativo del autoconcepto y ambos componentes de la Identidad Vocacional, independientemente de la relación con la Claridad del Autoconcepto.

Otra posibilidad a tener en cuenta es que el hecho de ser capaz de tomar una decisión en cuarto de secundaria afecte de forma positiva a la Autoestima más que en alumnos de quinto, ya que los alumnos que se muestren decididos y seguros de su elección en cuarto serían reforzados positivamente en mayor medida, tanto por sus pares como por sus educadores en el entorno escolar. Es importante 
considerar también el hecho de que en quinto de secundaria, la Autoestima esté siendo influida de forma más fuerte que en cuarto de secundaria por otras variables que no han sido consideradas en este estudio.

Al observar los puntajes significativamente mayores para Exploración y Compromiso obtenidos por los alumnos de quinto de secundaria cabe mencionar que, como estructura del sí-mismo, la identidad vocacional se encuentra en constante desarrollo (Holland, 1996; Marcia, 1980), por lo que es esperable que, siendo componentes de la Identidad Vocacional, tanto Exploración como Compromiso se tiendan a mostrarse más elevados con el aumento de la edad. Igualmente, estar en quinto de secundaria significa encontrarse en un período distinto, dada la cercanía con el término de la escuela, lo que originaría en muchos casos que sea necesario decidir y comprometerse con una opción de carrera, y que a su vez podría implicar un mayor involucramiento en tareas de exploración (Kalakoski y Nurmi, 1998). En la esta muestra, el grado de estudios en que se encuentra el individuo es un mejor indicador del nivel de Identidad Vocacional alcanzado, que la edad.

En cuanto a los resultados observados en la muestra dividida por sexo, es posible suponer que la autoestima de los adolescentes hombres se fundamente más en su capacidad para estar seguros de sus posibilidades de asumir un papel activo de producción y de comprometerse profundamente con un trabajo a través de una profesión, algo que no necesariamente sucedería en el caso de las mujeres, debido a patrones de género que llevan a otorgar una mayor importancia a la formación y la educación superior de los hombres como medio de subsistencia. Dichos patrones otorgarían en este caso una mayor libertad a las mujeres para explorar intereses, debido a una menor necesidad de enfocarse en el rendimiento económico en su carrera.

\section{Conclusiones}

El presente estudio ha contribuido al creciente cuerpo de investigaciones en temas de identidad vocacional y autoconcepto, aportando una mirada innovadora sobre la valía de la claridad del autoconcepto para el entendimiento del proceso de construcción de la identidad vocacional, algo que hasta el momento no había sido investigado directamente.

Es posible concluir que, en términos generales, la Identidad Vocacional, entendida como el conjunto dinámico de la Exploración y el Compromiso, se desarrolla de forma gradual en adolescentes, y tiende a desarrollarse hacia niveles más altos en el tiempo, y principalmente en relación a la transición a una etapa de estudios superiores. Esto concuerda con la perspectiva eriksoniana del desarrollo, según la cual la crisis de la adolescencia gira en torno al logro de la identidad frente a la potencial confusión de roles y de difusión del autoconcepto, y es un proceso que se da de forma progresiva (Erikson, 1974).

Asimismo, la Claridad del Autoconcepto se relaciona de forma significativa con la capacidad para tomar decisiones sobre la carrera, y a su vez, como una 
medida de la dimensión estructural de los contenidos del autoconcepto, podría explicar cierta variabilidad en la capacidad para decidir y comprometerse con la decisión que se tome, a diferencia de la dimensión afectivo-evaluativa, representada por el constructo de Autoestima. Teniendo esto en cuenta, es posible suponer que los casos en que existen problemas de la elección vocacional involucren aspectos estructurales del autoconcepto.

Por otro lado, se ha encontrado que la dimensión afectivo-evaluativa del autoconcepto proporciona una base fundamental para la resolución del proceso de exploración en la adolescencia, lo que constituye un aporte fundamental para la literatura en el tema, y concuerda con los resultados de estudios que investigaron la relación de aspectos de la identidad vocacional con medidas relacionadas con la estabilidad y coherencia del autoconcepto. En este sentido, la Autoestima podría presentar un valor predictivo de la variabilidad de la Exploración, a diferencia de la medida de la dimensión estructural, sumándole valor a las numerosas investigaciones previas enfocadas en la autoestima.

\section{Implicancias en la práctica}

En el trabajo con personas con dificultades para la elección vocacional es necesario tomar en cuenta el autoconcepto y sus distintas dimensiones, ya que contribuirán a un mejor entendimiento de las dificultades del individuo en el afrontamiento y la resolución de la crisis vocacional. Desde un punto de vista psicológico, el logro de un abordaje integral del problema de la elección profesional requerirá que se evalúe concretamente la situación del autoconcepto de un individuo y sus avances en cuanto al logro de una visión estable y clara de sí mismo. La orientación vocacional, como un proceso que movilice la capacidad de decisión autónoma del individuo mediante el esclarecimiento de su identidad (López, 2003) y la elaboración de su conflictiva interna (Katz, 1998), supone la necesidad de un conocimiento cuidadoso de la persona, primordialmente a través de su visión de sí misma (Rivas, 1998).

\section{REFERENCIAS}

Álvarez González, M. (1989). La madurez vocacional en el alumnado de secundaria (tesis doctoral, Universidad de Barcelona).

Álvarez González, M., Bisquerra, R., Espín, J. V. y Rodríguez Espinar, S. (2007). La madurez para la carrera en la educación secundaria. Evaluación e intervención. Sevilla: EOS.

Argumedo, D., y Romero, E. (2010). Escala de claridad del autoconcepto. Material no publicado.

Atienza, F. L., Moreno, Y., y Balaguer, I. (2000). Análisis de la dimensionalidad de la Escala de Autoestima de Rosenberg en una muestra de adolescentes valencianos. Revista de Psicología. Universitas Tarraconensis, 22(1-2), 29-42.

Barret, T. C., y Tinsley, H. E. (1977). Vocational self-concept crystallization and 
vocational indecision Journal of Counseling Psychology, 24(4), 301-307. doi:10.1037/0022-0167.24.4.301

Baumeister, R. F., and Twenge, J. M. (2003). The social self. En T. Millon, and M. J. Lerner (Eds.), Handbook of psychology: Personality and social psychology, Vol. 5 (pp. 327-352). Nueva York, NY: John Wiley and Sons.

Bedeian, A. G. (1977). The roles of self-esteem and $\mathrm{N}$ achievement in aspiring to prestigious vocations. Journal of Vocational Behavior, 11, 109-119. doi:10.1016/0001-8791(77)90021-5

Campbell, J. D. (1990). Self-esteem and the clarity of the self-concept. Journal of Personality and Social Psychology, 59, 538-549. doi:10.1037/00223514.59.3.538

Campbell, J. D., Trapnell, P., Heine, S., Katz, I., Lavallee, L., and Lehman, D. (1996). Self-concept clarity: Measurement, personality correlates, and cultural boundaries. Journal of Personality and Social Psychology, 70, 141-156. doi:10.1037/0022-3514.70.1.141

Campbell, J. D.; Assanand, S., and Di Paula, A. (2003). The structure of the selfconcept and its relation to psychological adjustment. Journal of Personality, 71(1), 115-140. doi:10.1111/1467-6494.t01-1-00002

Castañeda, A. K. (2013). Autoestima, claridad del autoconcepto y salud mental en adolescentes de Lima Metropolitana (tesis de licenciatura, Pontifica Universidad Católica del Perú).

Cohen, J. (1988). Statistical power analysis for the behavioral sciences. Nueva Jersey, NJ: Lawrence Erlbaum. doi:10.1002/bs.3830330104

Collins, W. A. and Laursen, B. (2004). Parent-adolescent relationships and influences. En R. M. Lerner, and L. Steinberg (Eds.), Handbook of Adolescent Psychology (2da ed.) (pp. 331-362). Nueva Jersey, NJ: John Wiley and Sons.

Demirutku, K. (2007). Parenting styles, internalization of values, and the selfconcept (tesis de Doctorado, Middle East Technical University).

Erikson, E. H. (1974). Identidad, juventud y crisis. Buenos Aires: Paidós.

Erikson, E. H. (2000). El ciclo vital completado. Barcelona: Paidós.

Fleming, J. S.; Courtney, B. E. (1984). The dimensionality of self-esteem: II. Hierarchical facet model for revised measurement scales. Journal of Personality and Social Psychology, 46(2), 404-421. doi:10.1037/0022-3514.46.2.404

Florenzano, R. (1993). En el camino de la vida. Santiago de Chile: Universitaria.

Gurung, R. A., Sarason, B. R., Sarason, I. G. (2001) Predicting relationship quality and emotional reactions to stress from significant-other-concept clarity. Personality and Social Psychology Bulletin, 27, 1267-1276. doi:10.1177/01461672012710003

Gushue, V. G., Clarke, C. P., Pantzer, K. M., and Scanlan, K. R. L. (2006). Selfefficacy, perceptions of barriers, vocational identity, and the career exploration behavior of latino/a high school students. Career Development Quarterly, 54(4), 307-317. doi:10.1002/j.2161-0045.2006.tb00196.x

Hirschi, A. (2009). Career adaptability development in adolescence: Multiple predictors and effects on sense of power and life satisfaction. Journal of Vo- 
cational Behavior, 74(2), 145-155. doi:10.1016/j.jvb.2009.01.002

Hirschi, A. (2011a). Vocational identity as a mediator of the relationship between core self-evaluations and life and job satisfaction. Applied Psychology: An International Review, 60(4), 622-644. doi:10.1111/j.1464-0597.2011.00450.x

Hirschi, A. (2011b). Career-choice readiness in adolescence: Developmental trajectories and individual differences. Journal of Vocational Behavior, 79(2), 340-348. doi:10.1016/j.jvb.2011.05.005

Hirschi, A. (2011c). Relation of vocational identity statuses to interest structure among swiss adolescents. Journal of Career Development, 38(5), 390-407. doi : $10.1177 / 0894845310378665$

Hirschi, A. (2012). Préparation à la transition de l'école obligatoire à la formation professionnelle. L'Orientation Scolaire et Professionnelle. doi : 10.4000/ osp. 3790

Hirschi, A., and Läge, D. (2007). Holland's secondary constructs of vocational interests and career choice readiness of secondary students. Journal of Individual Differences, 28(4), 205-218. doi:10.1027/1614-0001.28.4.205

Hirschi, A., and Läge, D. (2008). Using accuracy of self-estimated interest-type as a sign of career choice readiness in career assessment of secondary students. Journal of Career Assessment, 16(3), 310-325. doi: $10.1177 / 1069072708317372$

Holland, J. L. (1959). A theory of vocational choice. Journal of Counseling Psychology 6(1), 35-45. doi:10.1037/h0040767

Holland, J. L. (1996). Exploring careers with a typology. What we have learned and some new directions. American Psychologist 51(4), 397-406. doi:10.1037/0003-066X.51.4.397

Holland, J. L., Daiger, D. C., and Power, P. G. (1980). My Vocational Situation. Palo Alto, California: Consulting Psychologists Press.

Jara, L. (2010). Identidad vocacional en el tránsito del colegio a la universidad y en los primeros años de vida universitaria. Persona, 13, 137-157.

Josephs, R. A., and Markus, H. R. (1992) Gender and self-esteem. Journal of Personality and Social Psychology, 63(3), 391-402. doi:10.1037/00223514.63.3.391

Kalakoski, V., and Nurmi, J. E. (1998) Identity and educational transitions: Age differences in adolescent exploration and commitment related to education, occupation, and family. Journal of Research on Adolescence, 8(1), 29-47. doi:10.1207/s15327795jra0801_2

Katz, M. (2001). Orientación vocacional. Enfoque psicoanalítico. Psicoanálisis APdeBA, 23(2), 457-484.

King, L. A. (1958). Factors associated with vocational interest profile stability. Journal of Applied Psychology, 42(4), 261-263. doi:10.1037/h0043577

Lawrence, W. and Brown, D. (1976). An investigation of intelligence, selfconcept, socioeconomic status, race and sex as predictors of career maturity. Journal of Vocational Behaviour, 9, 43-52. doi:10.1037/h0043577

López, A. (2003). La orientación vocacional como proceso. Buenos Aires: Bonum 
Lubinski, D., Benbow, C. P., Ryan, J. (1995). Stability of vocational interests among the intellectually gifted from adolescence to adulthood: A 15year longitudinal study. Journal of Applied Psychology, 80(1), 196-200. doi:10.1037/0021-9010.80.1.196

Luyckx, K., Goossens, L., Soenens, B., Beyers, W., and Vansteenkiste, M. (2005). Identity statuses based on 4 rather than 2 identity dimensions: Extending and refining Marcia's paradigm. Journal of Youth and Adolescence, 34, 605-618. doi:10.1007/s10964-005-8949-x

Macovei, C. M. (2009). The forming of the vocational identity. Bulletin Scientific, $14(2) 74-79$.

Malone, P.(1989). The relationship of vocational identity to academic ability, locus of control and self-esteem (tesis de Maestría, University of Adelaide). Recuperado de http://digital.library.adelaide.edu.au/theses/09ARM/09armm257. pdf

Marcia, J. E. (1966). Development and validation of ego identity status. Journal of Personality and Social Psychology, 3(5), 551-558. doi:10.1037/h0023281

Marcia, J. E. (1967). Ego identity status: relationship to change in self-esteem, general maladjustment, and authoritarianism. Journal of Personality, 35(1), 119-133. doi:10.1111/j.1467-6494.1967.tb01419.x

Marcia, J. E. (1980). Identity in adolescence. En Adelson, J. (Ed.), Handbook of Adolescent Psychology (pp. 159-187). Nueva York, NY: Wiley Interscience.

Martínez, R. M., Tuya, L. C., Martínez, M. Pérez, A., Cánovas, A. M. (2009). El coeficiente de correlación de los rangos de Spearman. Caracterizacion. Revista Habanera de Ciencias Médicas, 8(2). Recuperado de http://scielo.sld.cu/ $\mathrm{pdf} / \mathrm{rhcm} / \mathrm{v} 8 \mathrm{n} 2 / \mathrm{rhcm} 17209 . \mathrm{pdf}$

Meeus, W. (1996). Studies on identity development in adolescence: An overview of research and some new data. Journal of Youth and Adolescence, 25, 569-598. doi:10.1007/BF01537355

Mullis, R. L., Mullis, A. K., and Gerwels, D. (1998). Stability of vocational interests among high school students. Adolescence, 33, 699-707.

Munson, W. W. (1992). Self-esteem, vocational identity, and career salience in high school students. Career Development Quarterly, 40(4), 361-368. doi:10.1002/j.2161-0045.1992.tb00343.x

Niece, D., and Bradley, R. (1979). Relationship of age, sex, and educational groups to career decisiveness. Journal of Vocational Behavior, 14, 271-278. doi: 10.1016/0001-8791(79)90055-1

Patton, W., Bartrum, D. A., and Creed, P. A. (2004). Gender Differences for Optimism, Self-esteem, Expectations and Goals in Predicting Career Planning and Exploration in Adolescents. International Journal for Educational and Vocational Guidance, 4, 193-209. doi:10.1007/s10775-005-1745-z

Rehberg, R., and Hotchkiss, L. (1979) Career counseling in contemporary U.S. high schools. En D. C. Berliner (Ed.), Review of research in education, Vol. 7 (pp. 92-147). Washington, D.C.: American Educational Research Association. 
Reina, M., Oliva, A., and Parra, A. (2010). Percepciones de autoevaluación: Autoestima, autoeficacia y satisfacción vital en la adolescencia. Psychology, Society, and Education, 2(1), 47-59.

Robbins, S. B., and Patton, M. J. (1985). Self-psychology and career development: Construction of the Superiority and Goal Instability Scales. Journal of Counseling Psychology, 32, 221-231. doi:10.1037/0022-0167.32.2.221

Rosenberg, M. (1965). Society and the adolescent self-image. Princeton, N.J.: Princeton University Press.

Rosenberg, M. (1973). La autoimagen del adolescente y la sociedad. Buenos Aires: Paidós.

Rottinghaus, P. J. (2007). Thirty-Year Stability and Predictive Validity of Vocational Interests. Journal of Career Assessment, 15(1), 5-22. doi: $10.1177 / 1069072706294517$

Santos, P. (2003). Goal instability, self-esteem, and vocational identity of high school Portuguese students. Análise Psicológica, 21(2), 229-238.

Savickas, M. L. (1985). Identity in vocational development. Journal of Vocational Behavior, 27(3), 329-337. doi:10.1016/0001-8791(85)90040-5

Savickas, M. L. (1990, marzo). Developing career choice readiness. Trabajo presentado en la Annual Convention of The American Association for Counseling and Development, Cincinnati.

Schwartz, S. J. (2001). The evolution of eriksonian and neo-eriksonian identity theory and research: A review and integration. Identity: An International Journal of Theory and Research, 1(1), 7-58. doi:10.1207/S1532706XSCHWARTZ

Skorikov, V. B. (2006). Adolescent career preparation, well-being, and distress. Trabajo presentado en el 19th Biennial Meeting of the International Society for the Study of Behavioral Development, Melbourne, Australia. Recuperado de http://www.docstoc.com/docs/83708019/418_20051029181 754 abs

Skorikov, V. B., and Vondracek, F. (1998). Vocational identity development: Its relationship to other identity domains and to overall identity development. Journal of Career Assessment, 6(1), 13-35. doi:10.1177/106907279800600102

Smith, M. C., and Dust, M. C. (2006). An exploration of the influence of disposal traits and appraisal on coping strategies in African American college students. Journal of Personality, 74, 145-174. doi:10.1111/j.1467-6494.2005.00372.x

Steffgen, G., Da Silva, M., and Recchia, S. (2007). Self-Concept Clarity Scale (SCSS): Psychometric properties and aggression correlates of a German version. Individual Differences Research, 5(3), 230-245.

Stinson, D. A., Wood, J. V., and Doxey, J. R. (2008). In search of clarity: Selfesteem and domains of confidence and confusion. Personality and Social Psychology Bulletin, 34, 1541-1555. doi:10.1177/0146167208323102

Strauser, D. R., Lustig, D. C., and Çiftçi, A. (2008). Psychological Well-Being: Its Relation to Work Personality, Vocational Identity, and Career Thoughts. The Journal of Psychology, 142(1), 21-35. doi:10.3200/JRLP.142.1.21-36 
Stringer, K. and J. Kerpelman (2010). Career identity development in college students. decision making, parental support, and work experience. Identity: An International Journal of Theory and Research, 10, 181-200. doi:10.1080 /15283488.2010.496102

Suh, E. M. (2002). Culture, identity consistency, and subjective well-being. Journal of Personality and Social Psychology, 83, 1378-1391. doi:10.1037/00223514.83.6.1378

Swanson, J. L. (1999). Stability and change in vocational interests. En Savickas, M. L. and Spokane, A. R. (Eds.), Vocational interests: Meaning, measurement, and counseling use (135-158). Palo Alto, CA: Davies-Black.

Taylor, K. M., and Betz, N. E. (1983). Applications of self-efficacy theory to the understanding and treatment of career indecision. Journal of Vocational Behavior, 22, 63-81. doi:10.1016/0001-8791(83)90006-4

Tracey, T. J. G., and Robbins, S. B. (2005). Stability of interests across ethnicity and gender: A longitudinal examination of grades 8 through 12. Journal of Vocational Behavior, 63, 335-364. doi:10.1016/j.jvb.2004.11.003

Tracey, T. J. G., Robbins, S. B., and Hofsess, C. D. (2005). Stability and change in interests: A longitudinal study of adolescents from grades 8 through 12 . Journal of Vocational Behavior, 66, 1-25. doi:10.1016/j.jvb.2003.11.002

Tosado, L. A. (2012). An exploratory study of a measure of vocational identity for spanish-speaking persons (tesis doctoral, University of Maryland).

Vondracek, F. W. (1992). The construct of identity and its use in career theory and research. The Career Development Quarterly, 41(2), 130-144. doi:10.1002/j.2161-0045.1992.tb00365.x

Vondracek, F. W., Schulenberg, J., Skorikov, V., Gillespie, L. K., Wahlheim, C. (1995). The relationship of identity status to career indecision during adolescence. Journal of Adolescence, 18, 17-29. doi:10.1006/jado.1995.1003

Vondracek, F. W., and Skorikov V. B. (1997). Leisure, school, and work activity preferences and their role in vocational identity development. The Career Development Quarterly, 45, 322-340. doi:10.1002/j.2161-0045.1997.tb00537.x

Waterman, A. S. (1988). Identity status theory and Erikson's theory: Commonalities and differences. Developmental Review, 8, 185-208. doi:10.1016/02732297(88)90003-2 